\title{
Efectos de la Covid-19 en el estrés laboral
}

Rildo Santos Bellido Medina

ORCID: https://orcid.org/ 0000-0002-8699-3490

rbellidome@unsa.edu.pe

Universidad Nacional de San Agustín de Arequipa

Arequipa, Perú

José Luis Aguilar Gonzales

ORCID: https://orcid.org/0000-0003-4662-4268

jaguilargo@unsa.edu.pe

Universidad Nacional de San Agustín de Arequipa Arequipa, Perú

\author{
María Elena Gamarra Castellanos \\ ORCID: https://orcid.org/ 0000-0001-6296-3601 \\ mgamarrac@unsa.edu.pe \\ Universidad Nacional de San Agustín de Arequipa \\ Arequipa, Perú
}

\author{
Karen Haydee Pastor Xespe \\ ORCID: https://orcid.org/0000-0002- \\ kpastorx@unsa.edu.pe \\ Universidad Nacional de San Agustín de Arequipa \\ Arequipa, Perú
}

\author{
Blanca Morales Palao \\ ORCID: https://orcid.org/0000-0001-9204-3400 \\ bmorales@unsa.edu.pe \\ Universidad Nacional de San Agustín de Arequipa \\ Arequipa, Perú
}

Recibido (29/04/21), Aceptado (14/05/21)

\begin{abstract}
Resumen: En el presente trabajo se plasman los resultados del análisis del estrés laboral en servidores públicos. Tomando en cuenta las variables sociolaborales presentes en el período de pandemia ocasionado por la Covid-19. Se expone el modelo demanda-control-apoyo social para determinar el estrés laboral en este contexto en dos gobiernos locales de la ciudad de Arequipa- Perú. Para este trabajo se consideró la participación de 286 colaboradores, y se utilizó un instrumento de recolección de información con 29 ítems que permitieron medir el estrés laboral. Los resultados muestran que existe un nivel medio de estrés en el trabajo, considerando que se trata de un trabajador activo y que en su trayecto al trabajo y durante las jornadas laborales, está expuesto a situaciones de riesgo. El estudio permitió concluir que las situaciones de salud actual son un condicionante del estrés en los servidores públicos.
\end{abstract}

Palabras Clave: Estrés laboral, Covid-19, situaciones de riesgo, situaciones de salud.

\section{Effects of Covid-19 on work stress}

Abstract: In the present work, the results of the analysis of work stress in public servants are reflected. Taking into account the socio-labor variables present in the pandemic period caused by Covid-19. The demand-control-social support model is exposed to determine work stress in this context in two local governments of the city of Arequipa-Peru. For this work, the participation of 286 collaborators was considered, and an information collection instrument with 29 items was used that allowed the measurement of work stress. The results show that there is an average level of stress at work, considering that it is an active worker and that on its way to work and during working hours, it is exposed to risky situations. The study allowed to conclude that current health situations are a conditioner of stress in public servants.

Keywords: Work stress, Covid-19, risk situations, health situations. 


\section{Introducción}

La pandemia actual producida por el Covid-19 ha repercutido en todos los escenarios de interacción de los seres humanos, es por ello, que el impacto en el ámbito laboral no ha sido ajeno, produciendo nuevos riesgos en el trabajo que afectan la salud y el bienestar laboral de los colaboradores, deteriorando la salud física, social y mental [1].

Las situaciones laborales en cualquier lugar del mundo, han traído importantes cambios de diferente índole, en principio gran cantidad de empresas han tenido que cerrar porque la situación de pandemia no les favorece para la venta de sus productos, por otro lado, el desempleo reducción de presupuesto ha ido creciendo cada vez más, y finalmente los empleos obligatorios están sujetos a los riesgos de salud actuales.

En ese sentido, los cambios inesperados y necesarios que se han presentado por esta pandemia en el sector laboral se perciben en las condiciones de trabajo, con altas demandas de trabajo para unos cuantos, despidos inmediatos, trabajo remoto y/o teletrabajo y la inseguridad contractual, dentro de los aspectos más relevantes, que han generado situaciones de angustia, temor, desconfianza que dañaron la salud mental de los colaboradores, incrementándose los niveles de estrés laboral [2], que además repercute en estrés social y familiar.

Es así como, tan sólo exponerse a altas exigencias emocionales, puede repercutir en actitudes poco favorables para el entorno de trabajo, como ausencia de liderazgo, deficiencia en las comunicaciones, además de relaciones sociales poco afectivas, que pueden influir en el rendimiento laboral [3]. A estos aspectos se suma el estrés inherente al trabajador, que puede estar sujeto a las diferentes situaciones familiares y las necesidades de protección de la familia.

En este trabajo se evaluó la situación laboral de los servidores públicos en la ciudad de Arequipa, en Perú. Considerando que se trata de un grupo laboral que debe mantener sus funciones aun en situación de pandemia. A pesar de que un importante número de trabajadores realiza actividades online, también existe un número considerable de colaboradores que deben asistir de forma presencial a sus funciones [4].

El estudio es transversal, descriptivo, no experimental [5], así mismo, el tiempo de recolección de información se realizó en los meses de enero y febrero del año 2021. Y para ello se consideraron 286 trabajadores, que realizan trabajo presencial y que deben enfrentar situaciones de riesgo de forma permanente.

El trabajo está compuesto de cuatro secciones, en la primera se han descrito los aspectos generales, en la segunda se incorporarán los fundamentos teóricos de esta investigación, en la tercera se explicarán las metodologías empleadas, finalmente en la última sección se expondrán los resultados, con su respectivo análisis y las conclusiones de este estudio.

\section{Desarrollo}

El estrés laboral es un fenómeno relacionado a múltiples factores de los riesgos psicosociales que pueden afectar la salud de los colaboradores [6], [7], así también, es la respuesta a estos factores que se presentan en el espacio laboral ocasionados por el inadecuado sistema laboral que aplican las organizaciones [8], [9].

En ese sentido, el estrés laboral es una situación que puede afectar a cualquier persona, sin distinción de raza, género o puesto que ocupa dentro de la organización. Está condicionado a numerosos factores [7], [9] y puede manifestarse de diversas formas, que pueden incluir situaciones rutinarias, descontento por las funciones laborales, exceso de tareas, presión de los supervisores, inconformidad salarial, ergonomía de los puestos de trabajo, entre otros factores como las relaciones personales, que finalmente el compendio de varias de estas, conducen a una baja productividad, riesgo en el trabajo, riesgo en la salud, y todos ellos afectan la producción empresarial [10],

Por tanto, el estrés producido en el trabajo en sentido estricto es definido como un síntoma general de adaptación, producido por los estímulos estresores, que afectan la condición de salud cuando permanecen constantes en el organismo [8], [11]. Los estímulos estresores son aquellas condiciones que causan reacciones emocionales, y que difieren de una persona a otra, ya que dependen de la personalidad y de las características propias de cada individuo [7].

El organismo recibe los estímulos estresores, y dependiendo de los mecanismos de control que pueda tener la persona, estos estímulos producirán poco nivel de estrés o mucho, o permanecerá un estrés constante o la atenuación del mismo (Fig.1). En cualquiera de los casos, la mejor solución es evitar el estímulo estresor [11].

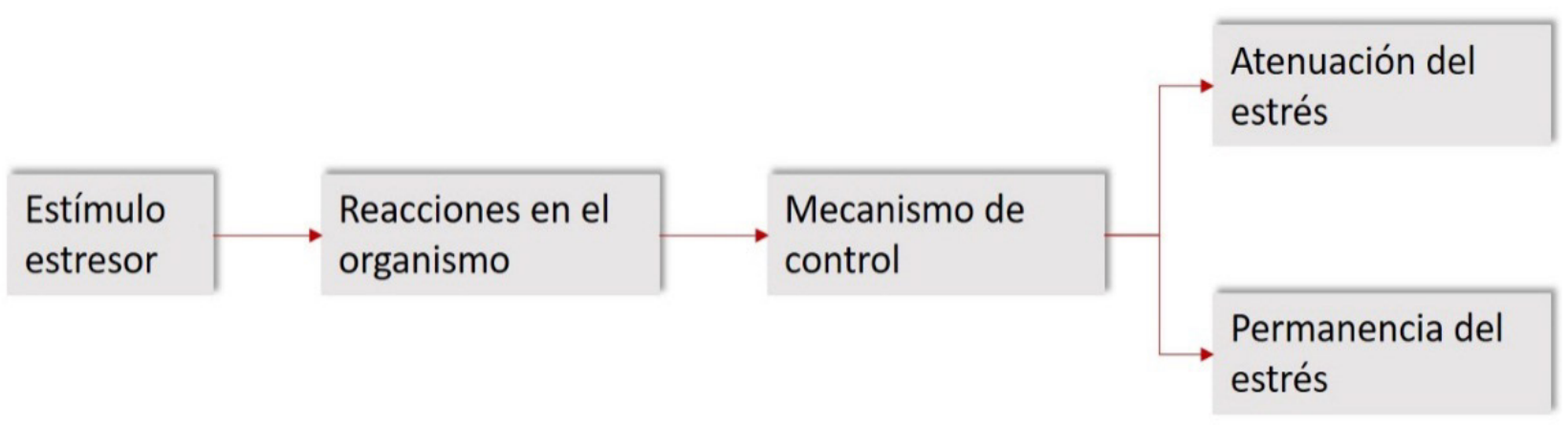

Fig. 1. Respuesta psicológica del estrés. 
Es deber de las organizaciones asegurar un ambiente laboral apropiado, generar los mecanismos de control de estrés, con políticas apropiadas y de respeto a los trabajadores, que promuevan un espacio de trabajo acorde a las necesidades pero valorando la salud de los trabajadores [12]. Sin embargo, la situación de pandemia no ha sido un factor suficiente para que las organizaciones mejoren las situaciones laborales, por el contrario, el problema de salud global atenúa las dificultades laborales, que incorporan en sus adversidades los problemas de salud sociales, de inseguridad, de riesgo.

En relación con el modelo propuesto por Karasek [13], señala tres aspectos importantes que se combinan para generar estrés como son la demanda-control-apoyo social, estos aspectos describen y explican todas las situaciones que ocurren en el trabajo donde los estresores son crónicos y ponen en total riesgo psicosocial a los colaboradores en el entorno laboral.

De esta manera la evaluación del modelo sugiere un estrés alto (fig.2), cuando las demandas y el exceso de control ocasionan ansiedad, fatiga, depresión y enfermedades físicas, además el modelo sugiere un estrés activo que comprende altas demandas y poco control que incide en la motivación y búsqueda de soluciones en la actividad laboral, un estrés bajo, donde las bajas demandas y el alto control no generan tensión en el trabajador y finalmente un estrés pasivo, en donde las bajas demandas y el bajo control ocasionan monotonía y falta de motivación en el empleado, impidiendo el desarrollo de las habilidades aprendidas [14], [7].

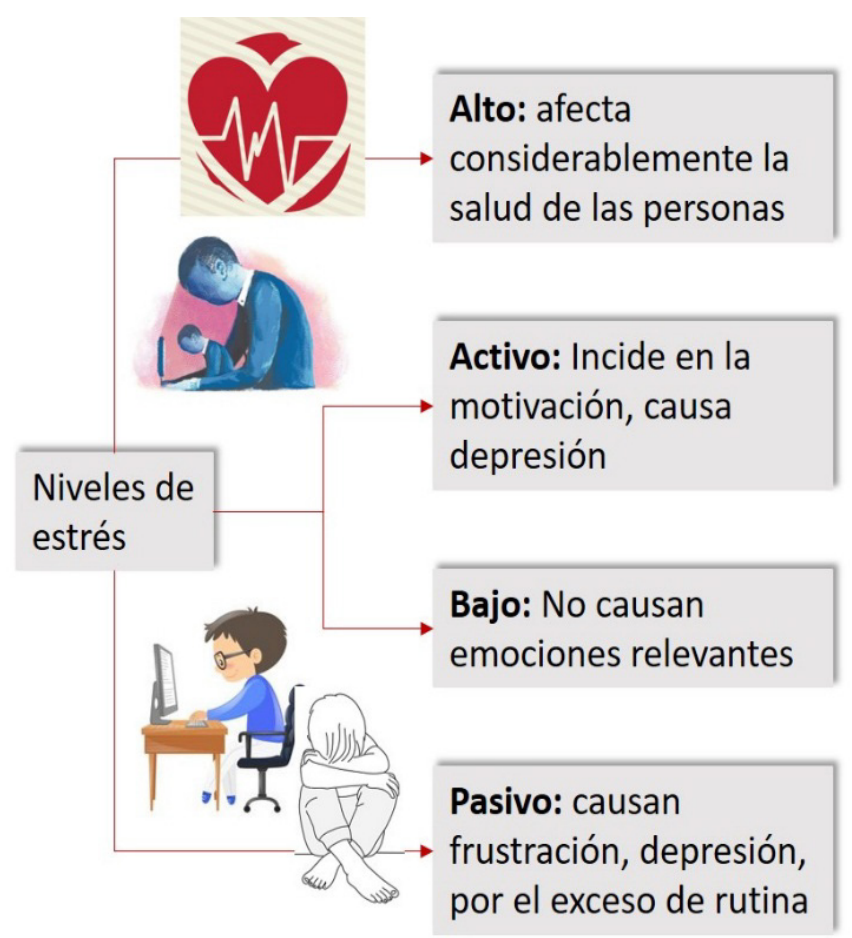

Fig. 2. Efectos del estrés en las personas, según las condiciones laborales.
Por otro lado, los estudios relacionados al estrés laboral en instituciones públicas peruanas se aproximan más al sector educación y salud, siendo los más relevantes, el estrés laboral en docentes peruanos [15] y el impacto del Covid-19 en la salud mental del personal de salud [16], sin embargo, no se encontraron estudios serios, actuales y de relevancia sobre estrés laboral en el actual contexto en instituciones gubernamentales en el Perú.

Conocer los efectos de la pandemia en los trabajadores de organismos gubernamentales del Perú, resulta un reto importante, que no solo involucra los aspectos de salud, sino las relacionales laborales de los trabajadores y sus empleadores. Este trabajo es el inicio de futuras investigaciones, donde se evalúen las condiciones sociales y laborales de las personas, con el fin de aportar con soluciones que favorezcan el estado emocional de los trabajadores y contribuyan a un buen desempeño de sus funciones, y en consecuencia a una alta productividad empresarial.

\section{Metodología}

Para este estudio, se incluyeron 286 colaboradores de la Ciudad de Arequipa, elegidos aleatoriamente, el rango de edad de los colaboradores se encontró entre los 21 a 60 años, de los cuales el $44.1 \%$ fueron mujeres y el $55.9 \%$ hombres. Se encontró que el $18.9 \%$ eran personas solteras, mientras que el $40.6 \%$ eran casados, el $32.2 \%$ eran convivientes, el $6.3 \%$ divorciados y el $2.1 \%$ viudos. Con respecto a las variables laborales de los colaboradores el 56.6\% mantenían la condición laboral de nombrados y $43.4 \%$ contrato CAS. Siendo empleados el $36.4 \%$ y personal obrero el $63.6 \%$. Con relación al tiempo de servicios se encuentran en promedio laborando entre 1 a 16 años en las instituciones evaluadas.

Para evaluar el estrés laboral se utilizó el modelo Demanda-Control-Apoyo Social (DC) de Karasek [17], que distingue, por un lado, las exigencias o demandas, en su mayoría de carácter psicosocial y, por otro, el control, entendido como el conjunto de recursos que el trabajador tiene para hacer frente a esas demandas. El instrumento consta de 29 ítems distribuidos en tres dimensiones que miden el control sobre el trabajo, las exigencias del trabajo y el apoyo social que recibe el colaborador.

Además, en el instrumento se incluyeron variables sociolaborales como edad, sexo, estado civil, condición laboral, centro de trabajo, tiempo de servicios. También se consignaron los datos del consentimiento informado. Para la versión a la muestra local se obtuvieron los niveles de confiabilidad de la escala mediante el método de consistencia interna con la prueba alfa de Cronbach [18] para el caso se obtuvo un $\alpha=0.882$ considerando que es un valor bueno para validar el cuestionario.

Para la realización de este trabajo se utilizó el consentimiento informado, de manera que tanto la institución involucrada como los participantes estuvieron de acuerdo en los procesos relacionados con la toma de datos y las características del estudio. De esta manera, se mantiene la confiabilidad de los datos respectivos. 


\section{IV.Resultados}

Los resultados de la investigación permiten la comparación del nivel de estrés laboral tomando en cuenta los factores sociolaborales como sexo, edad, centro de trabajo y condición laboral, por lo tanto, se exploraron los datos para analizar la distribución, la asimetría, curtosis y pruebas de normalidad, evidenciando que no existe distribución normal $(\mathrm{p}<.05)$. Se realizó el análisis descriptivo y el comparativo de la dedicación por el trabajo según el sexo, el grupo etario, la condición laboral y el centro de trabajo.

Para comparar dos muestras independientes se utilizó la U de Mann-Whitney siendo su tamaño del efecto, TE, cálculo de la probabilidad de superioridad, PSest, las normas interpretativas, No efecto (PSest $\leq=0.0$ ), pequeño (PSest $\geq$ $0,56)$, mediano (PSest $\geq 0,64)$ y grande (PSest $\geq 0,71)$ [19] [20]. La comparación de más de dos muestras independientes se realizó con la $\mathrm{H}$ de Kruskal Wallis, su tamaño del efecto utilizado fue $n^{2} \mathrm{H}$, eta cuadrada [21] siendo sus normas interpretativas $\mathrm{n}^{2} \mathrm{H} \geq 0.04$ mínima necesaria, $\mathrm{n}^{2} \mathrm{H} \geq 0.25$ modera$\mathrm{da}, \mathrm{n}^{2} \mathrm{H} \geq 0.64$ fuerte [22], y para establecer las correlaciones, se empleó la Rho de Spearman. Utilizándose para el análisis estadístico el programa SPSS 25.0.

La tabla 1 muestra el nivel de estrés en el trabajo, considerando las distintas dimensiones evaluadas, referentes al control y las exigencias y la manera en que el apoyo social favorece o no la situación de estrés.

Tabla 1. Nivel de estrés laboral y sus dimensiones de estudio

\begin{tabular}{ccccccccc}
\hline \multirow{2}{*}{ Nivel de estrés } & \multicolumn{2}{c}{ Estrés laboral } & \multicolumn{2}{c}{ Control } & \multicolumn{2}{c}{ Exigencias } & \multicolumn{2}{c}{ Apoyo social } \\
& $f(x)$ & $\%$ & $f(x)$ & $\%$ & $f(x)$ & $\%$ & $f(x)$ & $\%$ \\
\hline Bajo & 8 & $2.8 \%$ & 34 & $11.9 \%$ & 30 & $10.5 \%$ & 18 & $6.3 \%$ \\
Activo (medio) & 204 & $71.3 \%$ & 188 & $65.7 \%$ & 70 & $24.5 \%$ & 214 & $74.8 \%$ \\
Alto & 74 & $25.9 \%$ & 64 & $22.4 \%$ & 186 & $65.0 \%$ & 54 & $18.9 \%$ \\
Total & 286 & $100 \%$ & 286 & $100 \%$ & 286 & $100 \%$ & 286 & $100 \%$ \\
\hline
\end{tabular}

Se observa en la tabla 1 , que el $71.3 \%$ de colaboradores presentan nivel medio (activo) de estrés laboral, el 25.9\% nivel alto de estrés laboral y el $2.8 \%$ nivel bajo de estrés laboral. Con relación a las dimensiones de estudio, el $65.7 \%$ tiene nivel promedio de control sobre su trabajo, $22.4 \%$ alto control y el $11.9 \%$ presenta nivel bajo de control sobre el trabajo; así también, para el $65.0 \%$ el nivel de exigencias en el trabajo es alto, medio para el $24.5 \%$ y bajo en un $10.5 \%$; y según dimensión apoyo social el $74.8 \%$ recibe nivel medio de apoyo social, $18.9 \%$ nivel alto de apoyo social y $6.3 \%$ nivel bajo de apoyo social sobre todo por parte de los compañeros.
En la tabla 2 se muestra el nivel de estrés tomando en cuenta el sexo de las personas. Se comparan las diferencias estadísticamente significativas del estrés laboral según sexo del colaborador, de acuerdo con los resultados presentados en la tabla se observa que no existen diferencias significativas del estrés laboral según sexo, es válido afirmar que tanto hombres y mujeres presentan los mismos niveles de estrés laboral y que la variable pandemia también afectó a todos sin distinguir el sexo, por lo tanto, el nivel de afectación del estrés laboral se presenta de la misma manera en los colaboradores.

Tabla 2. Comparación del nivel de estrés laboral según sexo

\begin{tabular}{lccccc}
\hline \multicolumn{1}{c}{ Dimensiones } & $\begin{array}{c}\text { Hombres } \\
(n=160)\end{array}$ & $\begin{array}{c}\text { Mujeres } \\
(n=126)\end{array}$ & \multicolumn{2}{c}{ Estadigrafo } & \multirow{2}{*}{$P S_{\text {est }}$} \\
\cline { 2 - 5 } & Rango & Rango & $U$ & $p$ & \\
\hline Estrés laboral & 69.08 & 75.71 & 2286.5 & .342 & 0.01 \\
Control sobre el trabajo & 71.59 & 72.52 & 2487.0 & .893 & 0.03 \\
Exigencias del trabajo & 74.23 & 69.17 & 2341.5 & .466 & 0.01 \\
Apoyo social & 69.61 & 75.03 & 2329.0 & .426 & 0.01 \\
\hline
\end{tabular}

Nota: $\mathrm{n}=$ tamaño muestral; Rango = Rango Promedio; $\mathrm{U}=\mathrm{U}$ de Mann Withney; $\mathrm{p}=\mathrm{p}$ valor; PSest= Probabilidad de Superioridad (tamaño del efecto).

Las comparaciones del nivel de estrés mostradas en la tabla 3, según la edad, indican que no existen diferencias estadísticamente significativas en los colaboradores, es decir, que tanto los colaboradores jóvenes como los de mayor experiencia también experimentan los mismos niveles de estrés laboral. 
Tabla 3. Comparación del nivel de estrés laboral según edad

\begin{tabular}{|c|c|c|c|c|c|c|c|}
\hline \multirow{2}{*}{ Nivel } & \multirow{2}{*}{$\begin{array}{c}\text { De } 21 \text { a } 30 \\
\text { años } \\
(n=52) \\
\text { Rango }\end{array}$} & \multirow{2}{*}{$\begin{array}{c}\text { De } 31 \text { a } 40 \\
\text { años } \\
(n=104)\end{array}$} & \multirow{2}{*}{$\begin{array}{c}\text { De } 41 \text { a } 50 \\
\text { años } \\
(n=94)\end{array}$} & \multirow{2}{*}{$\begin{array}{c}\text { De } 51 \text { a } 60 \\
\text { años } \\
(n=36)\end{array}$} & \multicolumn{2}{|c|}{ Estadigrafo } & \multirow{2}{*}{$n^{2} H$} \\
\hline & & & & & $H$ & $p$ & \\
\hline Estrés laboral & 67.40 & 76.69 & 73.04 & 62.36 & 1.996 & $\begin{array}{c}.57 \\
3\end{array}$ & $\begin{array}{c}0.0 \\
1\end{array}$ \\
\hline Control sobre el trabajo & 71.31 & 75.36 & 72.63 & 61.67 & 1.500 & $\begin{array}{c}.68 \\
2\end{array}$ & $\begin{array}{c}0.0 \\
1\end{array}$ \\
\hline Exigencias del trabajo & 67.10 & 76.62 & 71.83 & 66.19 & 1.374 & $\begin{array}{c}.71 \\
2\end{array}$ & $\begin{array}{c}0.0 \\
1\end{array}$ \\
\hline Apoyo social & 65.08 & 77.19 & 74.71 & 59.92 & 3.446 & $\begin{array}{l}.32 \\
8\end{array}$ & $\begin{array}{c}0.0 \\
2\end{array}$ \\
\hline
\end{tabular}

Nota: $\mathrm{n}=$ tamaño muestral; Rango = Rango Promedio; $\mathrm{H}=\mathrm{H}$ de Kruskal Wallis; $\mathrm{p}=\mathrm{p}$ valor. $\mathrm{n} 2 \mathrm{H}=$ eta cuadrada (tamaño del efecto).

En la tabla 4 se comparan los niveles de estrés laboral según variables sociolaborales, con relación a la variable condición laboral, se encontraron diferencias estadísticamente significativas en el nivel de estrés laboral siendo los colaboradores con contrato CAS quienes presentan mayor estrés laboral (tamaño del efecto moderado), así mismo, en la dimensión exigencias del trabajo también se encontraron diferencias siendo los colaboradores CAS quienes sienten las exigencias del trabajo en mayor medida que los colaboradores nombrados (tamaño del efecto moderado), sin embargo, no se encontraron diferencias en las otras dimensiones (control sobre el trabajo y apoyo social).

Tabla 4. Comparación del nivel de estrés laboral según condición y ocupación laboral

\begin{tabular}{lcccccc}
\hline \multicolumn{1}{c}{ Dimensiones } & $\begin{array}{c}\text { Nombrado } \\
(n=160) \\
\text { Rango }\end{array}$ & $\begin{array}{c}\text { CAS } \\
(n=126) \\
\text { Rango }\end{array}$ & $\begin{array}{c}\text { Empleado } \\
(n=160) \\
\text { Rango }\end{array}$ & $\begin{array}{c}\text { Obrero } \\
(n=126) \\
\text { Rango }\end{array}$ & $(p) P S_{\text {est }}$ \\
\hline $\begin{array}{l}\text { Estrés laboral } \\
\begin{array}{l}\text { Control sobre } \\
\text { trabajo }\end{array}\end{array}$ & 71.91 & 72.12 & $(.031) 0.58$ & 70.23 & 75.11 & $(.021) 0.29$ \\
$\begin{array}{l}\text { Exigencias del trabajo } \\
\text { Apoyo social }\end{array}$ & 72.59 & 71.03 & $(.195) 0.02$ & 72.57 & 71.68 & $(.011) 0.18$ \\
\hline
\end{tabular}

Nota: $\mathrm{n}=$ tamaño muestral; Rango = Rango Promedio; $\mathrm{U}=\mathrm{U}$ de Mann Withney; $\mathrm{p}=\mathrm{p}$ valor; PSest= Probabilidad de Superioridad (tamaño del efecto).

De acuerdo a la variable ocupación laboral, es posible apreciar en la tabla 5, una correlación que muestra que si se encontraron diferencias estadísticamente significativas en el nivel de estrés laboral y sus dimensiones de estudio, en el nivel de estrés laboral son los colaboradores obreros quienes presentan mayores niveles de estrés que los colaboradores empleados debido a que realizan mayor trabajo presencial (tamaño del efecto pequeño), así también, los colaboradores obreros sienten en mayor medida las exigencias del trabajo que los empleados (tamaño del efecto pequeño); con respecto a la dimensión control sobre el trabajo son los colaboradores empleados quienes tienen mayor control de su trabajo que los obreros (tamaño del efecto pequeño), y según dimensión apoyo social, también son los colaboradores empleados quienes reciben mayor apoyo social que los obreros, sobre todo por parte de los compañeros y en menor medida por parte de los superiores. 
Tabla 5. Correlaciones del estrés laboral y las dimensiones de estudio

\begin{tabular}{ccccc}
\hline Rho Spearman & Control & Exigencias & Apoyo social & Estrés laboral \\
\hline Control & 1 & & & \\
Exigencias & $.625^{* *}$ & 1 & & \\
Apoyo social & $.418^{* *}$ & $.454^{* *}$ & 1 & 1 \\
Estrés laboral & $.808^{* *}$ & $.849^{* *}$ & $.610^{* *}$ & 1 \\
\hline
\end{tabular}

**. La correlación es significativa en el nivel 0,01 (2 colas).

Se mostraron altas correlaciones positivas directas tanto en el nivel de estrés laboral y control, así como, las exigencias del trabajo, y en la dimensión apoyo social y el estrés laboral la correlación es directa de nivel medio. Lo que demuestra que se cumple con el modelo control, demanda y apoyo social.

\section{Conclusiones}

Los resultados de acuerdo con el objetivo muestran que el nivel de estrés laboral en colaboradores de gobiernos locales durante el Covid-19 es de nivel medio, presentando altas demandas y poco control sobre el trabajo, esto probablemente por las condiciones laborales, además el riesgo y el miedo a contagiarse con el virus, aumenta los efectos de los estados emocionales, demostrando que las situaciones adversas por las que se atraviesan en la actualidad generan altos niveles de estrés.

Se ha demostrado que no existen diferencias estadísticamente significativas en la comparación del estrés laboral con las variables sexo y edad, sin embargo si se hallaron diferencias significativas en las variables laborales (condición y ocupación), debido a que las condiciones de trabajo difieren del personal nombrado al contratado CAS y sobre todo en los colaboradores empleados que realizan trabajo remoto frente a los obreros que trabajan de manera presencial exigiéndoles mayor productividad, aumentando la carga laboral y el nivel de exposición al virus, ya que los colaboradores trabajan a las afuera de la institución.

De acuerdo con el modelo de Karasek [17], según el primer principio demanda - control, se ha identificado que los colaboradores presentan altas demandas laborales y en nivel medio controlan su trabajo, por tanto, el modelo predice, riesgo de enfermedad relacionado con el estrés, y también predice las probabilidades de sufrir de estrés según los parámetros evaluados.

En el segundo principio demanda - apoyo social, el modelo predice que el nivel medio de apoyo que recibe el colaborador tanto de sus compañeros como superiores podría disminuir la habilidad del colaborador para hacer frente a una situación de estrés, por lo que, resulta indispensable que el ambiente laboral sea apropiado y exista un espíritu de compañerismo donde el apoyo a diferentes situaciones sea visible.

Y en el último principio demanda - control - apoyo social, tanto el control como el apoyo se ven influidos por los cambios de las organizaciones ante la situación de la pandemia, demostrándose que se presenta en mayor medida la exigencia. En ese sentido, Karasek, señala que un ambiente de trabajo estresante genera el desequilibrio entre demandas y respuestas, siendo la demanda el factor contingente que determina si un control escaso conduce a la pasividad o a la alta tensión psicológica. En tal sentido, el colaborador presenta mayores demandas y exigencias laborales frente a niveles medios de control y apoyo social, por lo tanto, se determina que es una persona potencialmente activa a incrementar el estrés laboral.

La situación de pandemia es una excusa para muchas organizaciones, que utilizan la situación de salud para desmejorar las condiciones laborales de sus trabajadores, y que no logran observar las posibles consecuencias que puede traer para la organización un trabajador en situación de estrés, como debilidad en las funciones que desempeña, poca proactividad, decaimiento, deficiencia, depresión, que finalmente impactaran en la producción y productividad de la institución.

Finalmente, se espera que el estudio haya sido de contribución académica para las diferentes disciplinas que abordan el estrés laboral, siendo un estudio relevante sobre todo por el aporte que se da en este contexto originado por el Covid-19. Además se espera que los empleadores consideren las consecuencias del estrés laboral, no solo en sus trabajadores sino en sus mismas instituciones.

\section{Referencias}

[1]L. Martínez, «Riesgos psicosociales y estrés laboral en tiempos de COVID-19: instrumentos para su evaluación,» Revista de Comunicación y Salud, vol. 10, n 2, pp. 301-323, 2020.

[2]L. Ortiz, «Estrés laboral en tiempos de pandemia,» UNA COMUNICA, 27 Mayo 2020. [En línea]. Available:https://www.unacomunica.una.ac.cr/index.php/mayo-2020/2970-estres-laboral-en-tiempos-de-pandemia. [Último acceso: 14 Enero 2021].

[3]P. Gil-Monte, «Algunas razones para considerar los riesgos psicosociales en el trabajo y sus consecuencias en la salud pública,» Revista Española de Salud Pública, vol. 83, n 2, pp. 169-173, 2019.

[4]G. I. Monzón Alvarez, M. Á. Pacheco Quico, A. R. Miaury Vilca, H. L. Pinto Pomareda y S. F. Torres Aza, «Riesgos laborales en personal de limpieza pública durante el Covid-19,» 
Universidad Ciencia Y Tecnología, vol. 25, nº 108, pp. 66-72, 2021.

[5]J. Calizaya, F. Zapata, G. Monzón, Y. Alemán, R. Bellido y F. Ceballos, La investigación cuantitativa en las ciencias sociales y algunas aplicaciones de interés científico, Quito: Editorial Autanabooks, 2021.

[6]J. Osorio y L. Cárdenas, «Estrés laboral: estudio de revisión,» Perspectivas Psicológicas, vol. 13, $\mathrm{n}^{\circ} 1$, pp. 81-90, 2017.

[7]F. Suárez, L. Rosales y O. Flor, La ingeniería de las emociones humanas, Quito: AutanaBooks, 2020.

[8]M. M. Chiang, G. R. Riquelme y P. A. Rivas, «Relación entre Satisfacción Laboral, Estrés Laboral y sus Resultados en Trabajadores de una Institución de Beneficencia de la Provincia de Concepción.,» Ciencia \& rabajo, vol. 20, nº 63, pp. 178-186, 2018.

[9]F. Suárez, L. Rosales y A. Lezama, La computación inteligente y los estados emocionales, Quito: AutanaBooks, 2019. [10]C. Mejia, J. Chacon, O. Enamorado, L. R. Garnica, S. Chacón y Y. García, «Factores asociados al estrés laboral en trabajadores de seis países de Latinoamérica,» Revista de la Asociación Española de Especialistas en Medicina del Trabajo, vol. 28, nº 3, pp. 204-211, 2019.

[11]F. Suárez, L. Ortiz y L. Rosales, «La psicología del estrés y su análisis desde la inteligencia artificial,» Espacios, vol. 45, no 41, p. 14, 2020.

[12]L. A. Álvarez-Silva y C. E. Espininoza-Samaniego, «Estrés laboral en trabajadores formales de la bahía de Guayaquil,» Espirales, vol. 3, n 24, pp. 77-88, 2019.

[13]R. Karasek, «Job demands, job decision latitude, and mental strain: Implications for job redesign,» Administrative Science Quarterly, vol. 24, pp. 285-309, 1979.

[14]J. M. Fernández-Arata y G. Calderón-De la Cruz, «Modelo Demandas-Control-Apoyo social en el estudio del estrés laboral en el Perú,» Revista Medica Herediana, vol. 28, pp. 281-282, 2017.

[15]O. Bada, R. M. Salas, E. F. Castillo, E. M. Arroyo y C. E. Carbonell, «Estrés laboral y clima organizacional en docentes peruanos,» MediSur, vol. 18, nº 6, pp. 1138-1144, 2020.

[16]A. Lozano-Vargas, «Impacto de la epidemia del Coronavirus (COVID-19) en la salud mental del personal de salud y en la población general de China,» Revista de Neuro-Psiquiatría, vol. 83, no 1, pp. 51-56, 2020.

[17]R. Karasek, Healthy work: stress, productivity, and the reconstruction of working, New York: Basic Books, 1990.

[18]D. Frías, Apuntes de consistencia interna de las puntuaciones de un instrumento de medida, Valencia: Universidad de Valencia, 2019.

[19]J.L. Ventura, «Tamaño del efecto para la U de Mann-Whitney: aportes al artículo de Valdivia-Peralta et al.,» Revista chilena de neuro-psiquiatría, vol. 54, nº 4, pp. 353-354, 2016. [20]R. J. Grissom, «Probability of the superior outcome of one treatment over another.,» Journal of Applied Psychology, vol. 79, no 2, pp. 314-316, 1994.

[21]M. Tomczak y E. Tomczak, «The need to report effect size estimates revisited. An overview of some recommended measures of effect size,» Trends Sport Sciences, vol. 1, $\mathrm{n}^{\circ} 21$, pp. 19-25, 2014.

[22]S. Domínguez, «Magnitud del efecto, una guía rápida,» Educación Médica, vol. 19, nº 4, pp. 251-254, 2018.

\section{RESUMEN CURRICULAR}

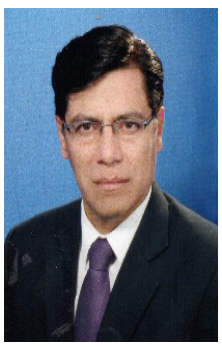

Bellido Medina Rildo Santos, Doctor en Psicología y Magíster en Gerencia Estratégica de Recursos Humanos. Docente auxiliar en el programa de estudios de Relaciones Industriales, Docente investigador UNSA- INVESTIGA y miembro del Instituto de Investigaciones Sociales de la Universidad Nacional de San Agustín, Arequipa, Perú

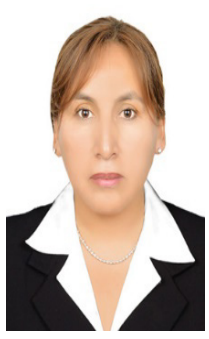

Gamarra Castellanos María Elena, Magíster en Ciencias con mención en Relaciones Industriales con mención en Gerencia Estratégica de Recursos Humanos, Grado de Doctor en Comunicación y Desarrollo; Licenciado en Ciencias de la Comunicación, Especialidad Relaciones Públicas. Docente Auxiliar en el programa de estudios de Relaciones Industriales en la Universidad Nacional de San Agustín, Arequipa, Perú

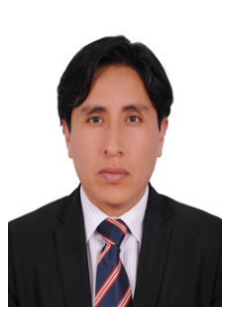

Aguilar Gonzales José Lu is, Doctor en Comunicación y Desarrollo, Magister en Ciencias de la Educación; con mención en Educación Superior, egresado de la Maestría de Gerencia Estrategia de Recursos Humanos; Licenciado en Ciencias de la Comunicación, Especialidad Relaciones Públicas. Docente ordinario a dedicación exclusiva en la especialidad de Relaciones Públicas y Periodis mo en la Universidad Nacional de San Agustín de Arequipa

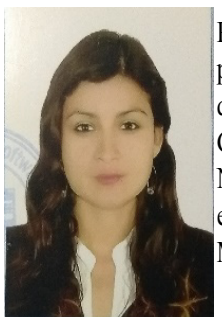

Pastor Xespe Karen Haydee, Licenciada en Trabajo Social por la Universidad Nacional de San Agustín de Arequipa, quinto superior durante los 5 años académicos. Magister en Gerencia Socialy de Recursos Humanos por la Universidad Nacional de San Agustín de Arequipa. Conciliadora extrajudicial y Conciliadora especializada en Familia por el Ministerio de Justicia y Derechos Humanos

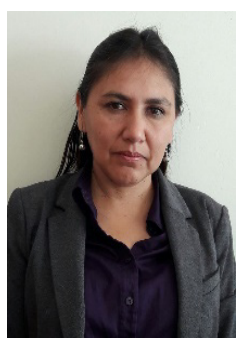

Morales Palao Blanca, Magíster en Ciencias, Relaciones Industriales con mención en Gerencia Estratégica de Recursos Humanos, Licenciada en Ciencias de la Comunicación, Especialidad Relaciones Públicas, Dip lomado en Recursos Humanos, Docente Auxiliar del programa de estudios de Ciencias de la Comunicación y miembro del Instituto de Investigaciones Sociales de la Universidad Nacional de San Agustín, Arequipa, Perú 